University of Texas at El Paso

ScholarWorks@UTEP

$5-2009$

\title{
Analysis of Information and Computation in Physics Explains Cognitive Paradigms: from Full Cognition to Laplace Determinism to Statistical Determinism to Modern Approach
}

Vladik Kreinovich

The University of Texas at El Paso, vladik@utep.edu

Roberto Araiza

The University of Texas at El Paso, raraiza@miners.utep.edu

Juan Ferret

The University of Texas at El Paso, jferret@utep.edu

Follow this and additional works at: https://scholarworks.utep.edu/cs_techrep

Part of the Computer Engineering Commons

Comments:

Technical Report: UTEP-CS-09-14

To appear in: Gordana Dodig-Crnkovic and Mark Burgin (eds.), Information and Computation, World Scientific.

\section{Recommended Citation}

Kreinovich, Vladik; Araiza, Roberto; and Ferret, Juan, "Analysis of Information and Computation in Physics Explains Cognitive Paradigms: from Full Cognition to Laplace Determinism to Statistical Determinism to Modern Approach" (2009). Departmental Technical Reports (CS). 40.

https://scholarworks.utep.edu/cs_techrep/40

This Article is brought to you for free and open access by the Computer Science at ScholarWorks@UTEP. It has been accepted for inclusion in Departmental Technical Reports (CS) by an authorized administrator of ScholarWorks@UTEP. For more information, please contact Iweber@utep.edu. 


\title{
Analysis of Information and Computation in Physics Explains Cognitive Paradigms: from Full Cognition to Laplace Determinism to Statistical Determinism to Modern Approach
}

\author{
Vladik Kreinovich ${ }^{1}$, Roberto Araiza ${ }^{1}$, \\ and Juan Ferret ${ }^{2}$ \\ ${ }^{1}$ Department of Computer Science \\ ${ }^{2}$ Department of Philosophy \\ University of Texas at El Paso \\ El Paso, Texas 79968, USA \\ emails vladik@utep.edu, \\ raraiza@miners.utep.edu, \\ jferret@utep.edu
}

\begin{abstract}
In this paper, we analyze the problem of prediction in physics from the computational viewpoint. We show that physical paradigms like Laplace determinism, statistical determinism, etc., can be naturally explained by this computational analysis. In our explanations, we use the notions of the Algorithmic Information Theory such as Kolmogorov complexity and algorithmic randomness, as well as the novel, more physics-oriented variants of these notions.
\end{abstract}

\section{Introduction}

Computability in theoretical computer science: traditional approach. Traditionally, the theoretical analysis of computations is mainly concentrated on analyzing which mathematical functions can be computed, which can be efficiently computed, etc.

Computability: a practical problem. It is desirable to take into account that from the practical viewpoint, computing mathematical functions (and solving precisely formulated mathematical problems) is an auxiliary task. 
One of the main objectives of computations is to predict the behavior of different processes in the real (physical) world. In view of this objective, it is important to distinguish between:

- general data and

- data that represents knowledge - i.e., that helps in this prediction.

It is also important to analyze what can be computably predicted and what cannot be predicted.

Predictability in physics: different paradigms. The understanding of what can be predicted has changed throughout the history of physics; see, e.g., [2]. Usually, a new paradigm appears when for some real-life processes, the previous paradigm turns out to be too optimistic; examples will be given below.

First paradigm: Full Cognition. In the beginning, the understanding was that science can potentially describe all the details of the process "from scratch". For example, Kepler was not only predicting how the planets move, he was also trying to predict the fate of princes by looking at the positions of the stars.

At present, we understand that it is possible to predict how the stars and the planets move, but it does not seem to be possible to predict human behavior and human fate. However, in Kepler's time, there seems to have been no understanding of this difference: science was supposed to be able to explain and predict everything. Yes, it is easier to predict how the planets move, it is more difficult to predict how people will behave, but both were considered legitimate science.

Similarly, now we know that it is not possible to make long-term (even yearly) weather predictions. However, even in the early 18 century, many weather almanacs were published that tried to predict weather for one (or more) years ahead - and these almanacs were actively bought by practicing farmers.

Limitations of the Full Cognition (FG) paradigm. The inability to predict such things as weather and human behavior led scientists to realize that a universal prediction "from scratch" is not always possible.

Laplace Determinism (LD). It turned out that while we cannot predict these phenomena based on general principles and ideas, we can often get reasonable predictions if we know the current state of the system.

For example, it is impossible to predict the fate of the newborn child based on the locations of stars and planets at the time when the child was born. However, when we observe the behavior of a person, when we find out his or her strengths, weaknesses, and behavior patterns, we can often reasonably well predict this person's fate. Similarly, when we observe the weather pattern in a given location, we can usually predict, e.g., that May will be dry and warm, that August will be rainy, etc. 
The idea that if we know the current state ("initial conditions") then we can predict the future behavior of the system was first explicitly formulated by Pierre-Simon Laplace, the 19 century mathematician and astronomer. Because of this, the corresponding paradigm is usually called Laplace Determinism.

Laplace Determinism: successes. Laplace Determinism has been the major paradigm of 19 century physics. Newton's mechanics, Maxwell's electrodynamics, many other physical theories - they are all formulated in terms of systems of differential equations, equations that enable us to go from the initial conditions to the predictions of the future states.

Even now, Laplace Determinism covers a significant portion of practical applications of physics.

Laplace Determinism: limitations. At the end of the 19 century, several new processes were discovered in which prediction turned out to be impossible. The most well known of these processes was radioactivity, i.e., the ability of atoms to spontaneously turn into other atoms.

All atoms of a radioactive material are absolutely identical, i.e., have practically identical initial conditions. So, from the viewpoint of the Laplace Determinism, they should all behave in exactly the same way - in particular, they should all undergo radioactive decay at the same time.

In reality, different atoms decay at different times, and all attempts to predict these times were unsuccessful.

Another limitation of Laplace Determinism comes from the analysis of decisions made by humans (and by other living beings). The famous Buridan's ass paradox considers a donkey placed between two absolutely identical bales of hay. Since these bales are absolutely identical, there is no reason for select one of them and not the other. Thus, a deterministic decision-making donkey will not be able to select one of these bales - and thus, the poor donkey may eventually starve to death. In practice, this will never happen - because a donkey would select one of the bales at random.

The need to take into account such random selections makes deterministic predictions impossible.

Statistical Determinism (SD). The physicists started collecting data about individual radioactive decay events. While they were unable to predict the time of individual events, they found out that the frequencies with which different atoms decay at different moments of time can be predicted.

In other words, while we cannot predict the exact future states, we can predict the probabilities of different future states.

At first, it was assumed that this need to restrict ourselves to statistical predictions was only typical of some exotic events like radioactivity. However, the successes of quantum physics have shown that a similar phenomenon holds for all physical predictions - at least those predictions in which we must take into account the properties of microobjects like atoms or elementary particles. 
Equations of quantum mechanics do not predict the values of the future location or velocity of a particle, they only describe the so-called wave function $\psi(x)$. Once we know the wave function, we can estimate the probability of finding a particle at a location $x\left(\right.$ as $\left.|\psi(x)|^{2}\right)$.

From the modern viewpoint, this need to consider probabilities is a wellknown textbook fact. However, in the 1920s and even in the 1930s, the idea that the Laplace Determinism paradigm must be replaced was very controversial: even Einstein famously said that he did not believe that God plays dice.

The paradigm in which, once we know the initial conditions, we can predict the probabilities of different future events, is usually called Statistical Determinism.

Statistical Determinism is still the prevailing paradigm in most areas of fundamental physics.

Limitations of Statistical Determinism and the emergence of a new paradigm. Starting with the 1960s, it turned out that for many real-life phenomena, even predicting probabilities is not always possible.

This phenomenon is best known for chaotic systems, i.e., systems in which a minor (practically non-observable) deviation in the initial conditions can lead to a drastic qualitative change in the future behavior of a system.

This phenomenon was first observed on the example of a simplified system of equations for predicting weather, and it has since been observed in numerous real-life phenomena.

Since even predicting probabilities is not always possible, the modern paradigm of physics - which we would call the Modern Approach (MA) - claims simply that if we know the initial conditions, then we can predict some information about the probabilities of different future states.

Physics paradigms: computational challenge. We have described the past and current physical paradigms. We have described them in a purely empirical way. Since these paradigms are about predictions - i.e., in effect, about prediction-related computations - it is desirable to understand these paradigms (and their relations and transitions) from the computational viewpoint.

What we do in this paper. In the paper, we will show that an appropriate computational analysis of predictability naturally explains the appearance of these different physical paradigms. We will also describe how this analysis can be used in working physics. As an example, we will give a recent unusual result from computational cosmology.

Comment. From the mathematical viewpoint, our analysis uses the notions of Algorithmic Information Theory such as Kolmogorov complexity and algorithmic randomness, as well as the novel, more physics-oriented variants of these notions. 


\section{Algorithmic Information Theory: How It Naturally Arises from Physical Paradigms}

The need to describe randomness. As we have mentioned, in Statistical Determinism, we predict the probabilities of different future states. For example, we predict the probabilities that an atom decays at different moments of time. We are unable to predict when each individual atom decays.

For example, if we start with a given set of $n$ atoms $a_{1}, \ldots, a_{n}$, and we know that at a certain moment of time, half of these atoms decay. We can describe the final state of this system by describing which atoms decayed and which did not. This can be naturally done by describing an $n$-bit sequence in which the $i$-bit is 1 if the $i$-th atom decayed and is 0 if it did not. For example, 101... means that the 1 st and the 3 rd atoms decayed, but the 2 nd atom did not. We cannot predict which of the $n$ atoms decayed. The actual sequence of decayed atoms is random, in the sense that all the information it contains is that half of the atoms decayed.

The fact that we cannot predict which atoms decay means that regular sequences like $010101 \ldots$ are not physically possible - because in such a sequence, we will be able to easily predict which atoms will decay and which atoms will not.

How can we describe this notion of randomness in precise terms?

Kolmogorov's original definition of randomness. In the $1960 \mathrm{~s}$, A. N. Kolmolgorov and his student P. Martin-Löf used the difficulty-to-predict as a formal definition of randomness.

They started by observing that when a binary sequence $s$ is regular, i.e., has the form $010101 . .$. , then even when this sequence is long, we can write a short (and simple) program $p$ that generates this sequence. For example, to generate a sequence consisting of 1,000 pairs 01 , we can write a simple for-loop:

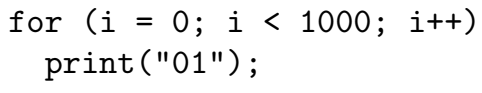

Vice versa, if a short program can generate a long sequence, this means that this sequence $s$ is regular.

The fact that a sequence is random means that it is not regular, i.e., that it is not possible to have a short program that generates this sequence.

Of course, we are dealing with finite sequences, and a finite sequence $s=$ $11001 \ldots$ can always be generated by a program that prints this sequence bit by bit:

print ("11001...");

However, if we use this idea to generate a sequence consisting of 1,000 symbols, we will need a program of the same length of 1,000 symbols (even a little bit longer, because we also need a print command).

Thus, the difference between regular sequences and random sequences is that 
- regular sequences can be generated by programs which are much shorter, while

- random sequences can only be generated by programs of approximately the same length.

To capture this difference, Kolmogorov and Martin-Löf defined Kolmogorov complexity $K(s)$ of a finite sequence $s$ as the shortest length of a program that generates the sequence $s$.

We can always generate a given sequence $s$ by simply printing it bit by bit. We have already mentioned that the length of this program is equal to the length len $(s)$ of the sequence $s$ plus a small number of bits $C$ needed to describe the print statement. Thus, the Kolmogorov complexity $K(s)$ of an arbitrary sequence $s$ - which is defined as the shortest length of the program that generates $s-$ cannot exceed the length len $(s)+C$ of this bit-by-bit program: $K(s) \leq \operatorname{len}(s)+C$.

Foe random sequences, no significantly shorter programs are possible. Thus, for a random sequence $s$, its Kolmogorov complexity $K(s)$ - the length of the shortest program for generating $s$ - cannot be much smaller than its length. This idea is behind the definition of randomness of a binary sequence.

Let $C$ be an integer. A finite binary sequenced is called $C$-random if $K(s) \geq$ $\operatorname{len}(s)-C$.

Comment. Strictly speaking, to make this definition precise, we need to fix a (universal) programming language, i.e., a programming language in which every algorithm can be programmed (like $\mathrm{C}$ or Java). However, it turned out that all the languages are, in some reasonable sense, equivalent: namely, the choice of a language only changes the length by a additive constant.

Beyond regular and random sequences: a useful consequence of Kolmogorov's formalization of randomness. In the previous paragraphs, we only considered two types of sequences: regular sequences and random sequences.

What about generic sequences? For an arbitrary sequence $s$, we can also describe its Kolmogorov complexity as the length of the shortest program $p$ that generates this sequence $s$. Once we know the program $p$, we can easily generate $s$ - by simply running a compiler from the corresponding programming language.

The important point is that, as we will show, the program $p$ is itself random in the Kolmogorov-Martin-Löf sense. Indeed, if we could generate $p$ by running a much shorter program $q$ with len $(q) \ll \operatorname{len}(p)$, then we would be able to generate $x$ from $q$ as well: by first generating the code $p$, and then by running this code to generate $x$. The fact that $p$ is the shortest program for generating $x$ means that $q$ cannot be much shorter than $p$-i.e., that the Kolmogorov complexity $K(p)$ must be approximately equal to the length len $(p)$ of this sequence $p$. 
By definition, sequence for which $K(p) \approx \operatorname{len}(p)$ are called random. Thus, $p$ is indeed a random sequence.

So, we arrive at a somewhat non-intuitive conclusion: that an arbitrary binary sequence $s$ can be obtained by applying a simple algorithm to some auxiliary random sequence $p$.

Comment. For a mathematician or for a physicist, the fact that an arbitrary sequence can be obtained by applying an appropriate algorithmic process to a random sequence may sound novel. While mathematics behind Kolmogorov's complexity is indeed reasonably novel, the possibility to represent an arbitrary signal as a combination of deterministic and random effects is well known and well established in statistical data and signal processing. There, the main objective - usually attainable - is to to find an approximate deterministic dependence for which the approximation error is purely random.

What we do in this paper. In this paper, we use the Kolmogorov complexity ideas to show that many physicists paradigms can be explained by the need for algorithmically feasible predictions.

\section{Explanation of Main Cognitive Paradigms of Physics}

Need for prediction: reminder. Our objective is to predict the state $s(t)$ of the Universe at future moments of time.

To be more precise, we would like to predict as much as possible about the state of the Universe at the future moments of time.

Ideal situation. In the ideal situation, we should have an algorithm that, given a future moment $t$, a location $x$, and the quantity $f$, would predict the value $f(x, t)$ of the quantity $f$ at the given location at the given moment of time.

This ideal situation corresponds to what we called full cognition.

Algorithm that uses measurement results. Since it turned out that no such universal algorithm is possible, the next natural idea is to use the initial conditions - i.e., the current values of the physical quantities - to predict the future state of the Universe. This is what we called Laplace Determinism.

Where is the beef? At this point, a reader may rightfully ask: what is the point of simply repeating the paradigms that we have already covered in the introduction? Up to now, there was indeed no point yet, but non-so-trivial consequences will start in the next paragraph. 
Interesting consequence: initial conditions should be random. What does this mean that we are unable to predict the state of the universe without using initial conditions? It simply means that initial conditions cannot be predicted "from scratch" - otherwise, we would be able to combine the two algorithms:

- algorithm for computing these initial conditions, and

- the algorithm for predicting the suture state of the world based on these initial conditions

and thus, get an (assumed impossible) algorithm for full cognition.

In algorithmic terms, what does it mean for a sequence to be unpredictable?

In Kolmolgorov's formalization, unpredictability simply means that the initial conditions are random.

Why is this consequence physically interesting? The fact that initial conditions are random may be an interesting mathematical observation, but does it have physical consequences? Yes, it does.

These physical consequences are related to the seeming contradiction between the physical equations and common sense about the direction of time.

From the purely mathematical viewpoint, Newton's laws do not change it we simply replace the original time coordinate $t$ with a new coordinate $t^{\prime}=-t$ - i.e., if we change the direction of time. If we videotape a purely mechanical motion and then show the motion backwards, the resulting motion will still make perfect sense.

However, when we turn from a system consisting of a few bodies to a system consisting of a large number of molecules, this time symmetry somehow disappears. Examples of this obvious (and mysterious) time asymmetry are well known.

For example, it is easy to observe that if we prepare a vessel with two separate compartments, place smoke in one compartment, leave clean air in another compartment, and then remove the separation, the gases will mix. The equations all remain time-invariant. Strictly speaking, if at the moment when the two gases have mixed, we simply invert the directions of all the velocities, we should see the same process reverting itself - and gases separating. A mathematician may say that this reversion is still physically possible but its probability is very low. However, a physicist would usually make a much stronger statement: the separation is simply not possible.

Let us give another example in which this impossibility is even more clear. If I drop a breakable cup on the hard floor, it will break into pieces. Theoretically, it may seem possible to imaging the reverse phenomenon: that the broken pieces magically fall back into place - but from the common sense viewpoint, everyone will agree that this magical recovery of the whole cup from pieces is not possible.

How do physicists explain this impossibility? Clearly this impossibility does not come from equations - since all the equations do not change if we simply replace $t$ with $-t$. A usual physicists' explanation is that the hypothetic cup 
recovery process is not stable. We never know the exact values of the initial locations and initial velocities, and if we change these initial conditions a little bit, the magic recovery of a broken cup will simply not occur.

In precise terms, what the physicists are saying is that for a cup to recover, we need to have very special, very well-defined initial conditions. However, since there are many unpredictable effects, the actual initial conditions cannot be predictable and exact.

In other words, what the physicists are saying is that it is not sufficient to describe the equations describing physical dynamics, we must also postulate that the initial conditions are random.

Our claim is that there is actually no need for a special new postulate - the very fact that we went from Full Cognition to Laplace Determinism means, in effect, that the initial conditions should be random.

Towards explaining other physical ideas within the Laplace Determinism paradigm. The fact that the randomness of initial conditions seems to follow naturally from our analysis of cognition makes us wonder that maybe other physical ideas within Laplace Determinism can be also be naturally explained by a similar analysis.

Indeed, the general idea of Laplace Determinism is that we predict the values $f(x, t)$ of different physical quantities $f$ at difference locations $x$ and at different future moments of time $t$ based on the initial conditions $g\left(x, t_{0}\right)$. In the worstcase scenario,

- to predict each value $f(x, t)$, we need to have full information about all the initial conditions, i.e., to have all the values $f\left(y, t_{0}\right)$ corresponding to all possible spatial locations $y$;

- each quantity can attain all possible numbers as its values, so, e.g., to get the value with accuracy $2^{-k}$, we need to actually generate all $k$ bits from this sequence;

- finally, once we have succeeded in predicting the value of one physical quantity $f$ at a certain location, this prediction will not in any way help in predicting the values of other quantities at different spatial locations.

The resulting computations require a large amount of input (all the initial conditions), produce the large size output (all bits need to be produced), and cannot utilize other previous predictive computations. In this worst case, the success of these predictive computations is thus in doubt. To make successful predictions, it is therefore reasonable to look for physical systems in which at least some of these complexities are relaxed.

Let us describe possible relaxations and let us show that they indeed correspond to fundamental physical ideas.

Relaxing input complexities: the notion of causality. Let us first consider what will happen is we relax the need for the value $f(x, t)$ to depend on 
the initial values $g\left(y, t_{0}\right)$ for all possible spatial locations $y$. Relaxing this need means that we assume that the value $f(x, t)$ of a physical field only depends on the values $g\left(y, t_{0}\right)$ for some spatial locations.

The values of $g\left(y, t_{0}\right)$ at other spatial locations $y$ do not affect the values $f(x, t)$ at all.

Since physical fields are interconnected, it is reasonable to expect that since the value $f(x, t)$ of the physical field $f$ does not depend on $g\left(y, t_{0}\right)$, the values of all other physical fields at the location $x$ at moment $t$ are also not affected by the values $g\left(y, t_{0}\right)$. Thus, this limitation does not depend on the specific fields, it depends only on the space-time points $(x, t)$ and $\left(y, t_{0}\right)$.

Thus, we have space-time moments $(x, t)\left(t>t_{0}\right)$ which are affected by the moments $\left(y, t_{0}\right)$ and space-time moments which are nor affected. In other words, we naturally arrive at the notion of causality, the notion that - in this spacetime form - only appeared in the mainstream physics in the early 20 century, with Einstein's Special Relativity Theory.

What we have shown is that from the algorithmic viewpoint, this notion can be very naturally derived.

Relaxing output limitations: bounds on physical quantities, quantization, conservation laws. The output complexity comes from the fact that, in general, all real values are possible for each physical quantity. In the worst case scenario,

- arbitrarily large and arbitrarily small values are possible, and

- once two real values are possible, all intermediate values are possible too.

Thus, to relax these limitations, it is reasonable to consider the possibility that

- there are bounds on the values of physical properties - from above and/or from below, and

- not all intermediate values are possible, there is only a discrete set of possible values.

Such possibilities are well known in physics. For example, there are bounds on the possible values of the velocities (bounded by the speed of light), distance (bounded by the size of the Universe), entropy's rate of change (bounded by 0 from above), etc.

It is also well known that some quantities like electric charge can only take values from a certain discrete of values.

For some quantities, possible future values are limited to a single value these are properties described by conservation laws such as energy conservation.

Relaxing the independence limitations: the emergence of symmetry. Finally, the relaxation of the third complication - that predicting one values $f(x, t)$ does not help in predicting other values - means that once we know the 
value $f(x, t)$, this will help us predict the values of some other quantities at some other spatial locations and moments of time.

The possibility to use the values at some points to make predictions about the values at other points is related to the notion of symmetry, one of the most fundamental notions of modern physics.

These physical consequences are not always independent. In our informal description, all these physical consequences seem independent from each other. However, a detailed analysis has shown that they these consequences are actually interrelated.

For example, an accurate Kolmogorov complexity-based formalization of the idea that initial conditions should be random leads to the need to bound the values of physical quantities; see, e.g., [6].

To be more precise, this interrelation is based not only on the original notion of Kolmogorov randomness but also on it physics-related modification. This modification $[3,6]$ is based on the fact that according to physicists, events with sufficiently small probabilities cannot occur. This means, in particular, that once we have a definable sequence of events $A_{1} \supseteq A_{2} \supseteq \ldots \supseteq A_{n} \supseteq \ldots$ with an empty intersection $\cap A_{n}=\emptyset$, we have $p\left(A_{n}\right) \rightarrow 0$ and therefore, for some $N$, this probability becomes so small that this event does not occur.

We can thus require that the set $E$ of actually possible events has the following property: for every definable monotonic sequence $A_{n} \supseteq A_{n+1}$ with the empty intersection, there exists an integer $N$ for which $A_{N} \cap E=\emptyset$.

To show that this definition leads to boundedness of a physical quantity $q$, we can simply take, as $A_{n}$, the set of all the events in which the value of $q$ is outside the interval $[-n, n]:|q|>n$. Clearly, this sequence is monotonic, and the intersection of all the sets $A_{n}$ is empty: since a real number cannot be larger than all possible integers $n$.

Thus, there exists a value $N$ for which no events from the set $A_{N}$ can occur. By definition of the event $A_{N}$, this means that values $q$ with $|q|>N$ are impossible - and thus, that all the physical values of the quantity $q$ are bounded by the bound $N$.

Comment. This argument sounds simple - and it is simple. However, this simplicity is based on a rather complex proof that the above requirement on the set $E$ of actual events is indeed physically possible; for details of this proof, see, e.g., [6] and references therein.

What if we still cannot predict everything? In the previous paragraphs, we describe possible physical ideas that can be helpful in predicting the future state of the physical world - i.e., in effect, the future values of different physical quantities.

But what if, even with all these physical ideas, we are still unable to make predictions? According to our Kolmogorov complexity analysis, this would simply mean that the resulting values $f(x, t)$ cannot be algorithmically determined 
based on the initial conditions - i.e., that there are some random events that also determine these future values.

In such situation, we cannot predict the actual values of the future quantities, but we can predict the probabilities of different values. This is the situation that we called Statistical Determinism.

Beyond Statistical Determinism. At first glance, according to our analysis, the algorithms operating on random sequences cover all possible measurement results. Since this representation corresponds to Statistical Determinism, why do we need to go beyond this physical paradigm?

The main reason is that in the original Kolmogorov's analysis, he only took into account the length of the corresponding program, but not its running time. For physical predictions, running time can be crucial: if we can predict the future, but these predictions will continue way after these events occur, then these predictions are useless. Alas, this possibility is not purely theoretical: there are many important real-life prediction problems - such a predicting where a tornado will go - for which the only known predicting algorithms require running time which goes way past the actual tornado motion.

For such algorithms, even probabilities are difficult to predict. A typical example of such situations are chaotic systems. Chaotic systems are usually deterministic, there is a simple program generating this sequence - but the actual predictions are nevertheless not computationally possible.

In mathematical terms, these situations are described by resource-bounded Kolmogorov complexity, versions of Kolmogorov complexity that take into account not only the length of the program, but also its running time - and maybe other characteristics like the number of processors needed to run the corresponding algorithms. At present, applications of resource-bounded Kolmogorov complexity to physics are much less developed that for the traditional Kolmogorov complexity. We hope that future developments in this area will pour some light on what we called Modern Approach paradigm of physics.

\section{Case Study: A Recent Cosmological Break- through as an Example of a Successful Pre- diction}

Prediction in cosmology: a brief description of the problem. Cosmology studies how the Universe as a whole evolves, it studies the large-scale structure of the Universe.

For cosmology, we can also formulate a usual prediction problem: how can we predict the future state of the Universe? What will happen in the next ten billion years? These questions are of great fundamental importance. However, because of the large-scale structure of the corresponding processes, these predictions cannot be directly verified - unless we are willing to wait for several more billion years. 
What we can try to "predict" - and where we can check our predictions is what happened in the past. We have numerous observations about the past, for two reasons:

- first, observations from distant galaxies actually come from the distant past, simply due to the fact that, e.g., to traverse a distance of one billion light years, light has to travel one billion years;

- second, we often observe physical processes that have "frozen" in time, remain largely unchanged since the distant past; the most well known phenomenon of this type is $3 \mathrm{~K}$ radiation that comes from the early stage of the Universe; there are other such phenomena as well.

Prediction in cosmology: challenges. One of the main problems with prediction in general is that for many physical systems, small deviations increase exponentially with time. Thus, with the advent of time, even the small uncertainty in the initial condition translates into an enormous uncertainty at prediction time, so enormous that the prediction becomes practically useless.

Such a situation occurs in meteorology, where, because of this exponential growth, we are unable to predict weather for periods exceeding a few days or weeks. This problem is especially serious in cosmology, where dynamics involves time periods of more than ten billion years.

The fact that in cosmology, fluctuations grow exponentially, is well known. Indeed, from observing the $3 \mathrm{~K}$ radiation, the remainder of the original state of the world, we can conclude that at that time, the state was almost perfectly isotropic and homogeneous, inhomogeneities were extremely small, barely distinguishable. However, these small homogeneities have led to the whole modern structure of the Universe, with all the rich structure of galaxies, clusters, stars, planets, etc.

From the purely theoretical viewpoint, in cosmology, we are in an enviable situation: in addition to knowing the state $s\left(t_{0}\right)$ of the Universe at the current moment of time $t_{0}$, we also know (via the $3 \mathrm{~K}$ radiation) the state at a moment $t_{r}$ close to the beginning of the Universe. We know how the Universe evolves, so theoretically, we can use both known states as initial conditions to predict the phenomena at some past times $t$ :

- we can start with the current state $s\left(t_{0}\right)$ and integrate the known dynamical equations back into the past, or

- we can start with the original state of the Universe $s\left(t_{r}\right)$, and integrate the same dynamical equations into the future.

However, in practice, due to the exponential growth of fluctuations, both methods do not lead to reasonable predictions.

A recent breakthrough: description. A few years ago, a group of cosmologists and mathematicians came up with a novel idea: to use both the current 
state $s\left(t_{0}\right)$ and the original state $s\left(t_{r}\right)$ when predicting the past states $s(t)$. This idea has led to successful predictions: specifically, at the large-scale level, they succeeded in using the $3 \mathrm{~K}$ state and current locations of the galaxy clusters to "predict" (reconstruct) the velocities of these clusters (see, e.g., [1, 4, 8]; see also [5]).

A recent breakthrough: methodological challenge. From the physical viewpoint, the above result is spectacular. However, this result raises a natural methodological question: how did the above idea overcome the challenge of exponential growth of fluctuations, a challenge that seems to make all longterm predictions impossible?

What we do in this section. In this section, we show that the above novel approach can indeed lead to successful predictions, even in situations with exponentially growing fluctuations.

Thus, in other physical areas, exponential growth of fluctuations does not necessarily means that predictions are completely impossible: when we supplement the current state $s\left(t_{0}\right)$ with the additional knowledge about the past, we can indeed get very good predictions.

Exponential growth of fluctuations: reminder. Before we present our explanations, let us recall where the exponential growth of fluctuations comes from.

We want to trace how small deviations $x(t)$ from a current state change with time. In general, the change in these deviations is described by a dynamical equation $\frac{d x}{d t}=f(x)$ for an appropriate function $f(x)$. Since the deviations are small, we can expand the function $f(x)$ in Taylor series and keep only linear terms in this expansion. Thus, we get a system of equations $\frac{d x}{d t}=a+A x$ for some appropriate vector $a$ and a matrix $A$.

Since $x$ are deviations from the given state, if we start with the current state (i.e., with $x\left(t_{0}\right)=0$ ), we should end up with the current state as well, i.e., with $x(t)=0$ for all $t$. Thus, the value $x(t)=0$ corresponding to the unperturbed state must satisfy the above equations. Substituting $x(t)=0$ into these equations, we conclude that $a=0$. Thus, the dynamical equations take the following form

$$
\frac{d x}{d t}=A x
$$

Solutions to these equations are well known. To describe these solutions, we can use the eigenvectors of the matrix $A$. In the non-degenerate case, when all the eigenvalues of the matrix $A$ are different, we can simplify the above system if we use the eigenvectors $e_{i}$ of the matrix $A$, i.e., unit vectors for which $A e_{i}=\lambda_{i} \cdot e_{i}$ for the corresponding eigenvalues $\lambda_{i}$. 
It is well known that the eigenvalues form a basic, in the sense that an arbitrary vector $x(t)$ can be described as a linear combination of these eigenvectors:

$$
x(t)=c_{1}(t) \cdot e_{1}+\ldots+c_{n}(t) \cdot e_{n} .
$$

Substituting this expression into the above dynamical system and using the fact that $A x_{i}=\lambda_{i} \cdot x_{i}$, we conclude that

$$
\sum_{i=1}^{n} \frac{d c_{i}(t)}{d t} \cdot e_{i}=\sum_{i=1}^{n} \lambda_{i} \cdot c_{i}(t) \cdot e_{i}
$$

Since the vectors $e_{i}$ form a basis, the fact that the vectors on the left-hand side and on the right-hand side of the above equation coincide means that the coefficients at $e_{i}$ at both sides must coincide too, i.e., that for every $i$, we must have

$$
\frac{d c_{i}(t)}{d t}=\lambda_{i} \cdot c_{i}(t)
$$

This simple differential equation has a known solution $c_{i}(t)=C_{i} \cdot \exp \left(\lambda_{i} \cdot t\right)$ for some constant $C_{i}$. Thus, the general solution to the above dynamical system has the form

$$
x(t)=\sum_{i=1}^{n} C_{i} \cdot \exp \left(\lambda_{i} \cdot t\right) \cdot e_{i} .
$$

When we know the state $x\left(t_{0}\right)$, we thus know the coefficients $c_{i}\left(t_{0}\right)$. Thus, in principle, from the value $c_{i}\left(t_{0}\right)=C_{i} \cdot \exp \left(\lambda_{i} \cdot t_{0}\right)$, we can conclude that $C_{i}=c_{i}\left(t_{0}\right) \cdot \exp \left(-\lambda_{i} \cdot t_{0}\right)$ and thus, that for every other moment $t$, we have

$$
c_{i}(t)=C_{i} \cdot \exp \left(\lambda_{i} \cdot t\right)=c_{i}\left(t_{0}\right) \cdot \exp \left(\lambda_{i} \cdot\left(t-t_{0}\right)\right)
$$

Theoretically, this is the desired predicting formula, but in practice, predicting is difficult:

- when we predict from the moment $t_{0}$ into the past, the factor

$$
\exp \left(\lambda_{i} \cdot\left(t-t_{0}\right)\right)
$$

grows exponentially for eigenvalues with negative real part; thus, negligible uncertainty in the current values $c_{i}\left(t_{0}\right)$ lead to huge uncertainty in the past state;

- on the other hand, when we predict from the moment $t_{r}$ into the future, the factor $\exp \left(\lambda_{i} \cdot\left(t-t_{r}\right)\right)$ grows exponentially for eigenvalues with positive real part; thus, negligible uncertainty in the current values $c_{i}\left(t_{r}\right)$ lead to huge uncertainty in the future state.

For cosmological systems it is well known that such an exponential increase indeed occurs - meaning that the corresponding matrix has both eigenvalues with positive real parts and eigenvalues with negative real parts. 
Comment. In the degenerate case, the general solution is similar, with the exception that we can also have additional solutions of the type $t^{k} \cdot \exp \left(\lambda_{i} \cdot t\right)$. In this case, the dynamic equations are slightly different, but the exponential growth of fluctuations remains.

What happens when we use both the current state and the past state for predictions. If we use both the current state $x\left(t_{0}\right)$ and the past state $x\left(t_{r}\right)$ for predictions, then, for each $i$, we have both values $c_{i}\left(t_{0}\right)$ and $c_{i}\left(t_{r}\right)$. In this case, for a given moment of $t$ between $t_{r}$ and $t_{0}$, we can predict the values $c_{i}(t)$ as follows:

- for the eigenvalues $\lambda_{i}$ with negative real parts, we can predict the values $c_{i}(t)$ as $c_{i}(t)=c_{i}\left(t_{r}\right) \cdot \exp \left(\lambda_{i} \cdot\left(t-t_{r}\right)\right)$;

- on the other hand, for the eigenvalues $\lambda_{i}$ with non-negative real parts, we can predict the values $c_{i}(t)$ as $c_{i}(t)=c_{i}\left(t_{0}\right) \cdot \exp \left(\lambda_{i} \cdot\left(t-t_{0}\right)\right)$.

Once we know the values $c_{i}(t)$, we can reconstruct the state $x(t)=\sum_{i=1}^{n} c_{i}(t) \cdot e_{i}$.

In both cases (of eigenvalues with negative real parts and of eigenvalues with non-negative real parts), the absolute values of the coefficients at $c_{i}\left(t_{0}\right)$ and $c_{i}\left(t_{r}\right)$ remain bounded by 1 . Thus, fluctuations do not grow with time, and we can predict the values at all intermediate moments of time $t$ with the same accuracy with which we know the current and the past states.

Thus, prediction based on past and future values is indeed possible.

Comment. The difference between predicting based only on the current state or only on the past state - and the prediction based on both states - is similar to the known difference between extrapolation and interpolation:

- extrapolation means predicting values outside the interval on which the values are known, while

- interpolation means predicting values inside the interval on which the values are known - filling he gaps.

It is known that interpolation is often practically possible, while extrapolation is often not possible - especially if we want to extrapolate far away from the known values. This is exactly what we observe now:

- prediction only from the present or only from the past - an analogue of extrapolation - is, for cosmology, not practically possible;

- on the other hand, prediction of the values $x(t)$ based on the values $x\left(t_{r}\right)$ and $x\left(t_{0}\right)$ for which $t_{r}<t<t_{0}-$ an analogue of interpolation - is practically possible. 


\section{Acknowledgments}

This work was supported in part by the National Science Foundation grant HRD-0734825, by Grant 1 T36 GM078000-01 from the National Institutes of Health, and by Grant MSM 6198898701 from MŠMT of Czech Republic.

\section{References}

[1] Y. Brenier, U. Frisch, M. Hnon, G. Loeper, S. Matarrese, R. Mohayaee, and A. Sobolevskii, "Reconstruction of the early Universe as a convex optimization problem", Monthly Notices of the Royal Astronomical Society, 2003, Vol. 346, pp. 501-524.

[2] R. Feynman, R. Leighton, and M. Sands, The Feynman Lectures on Physics, Addison Wesley, Boston, Massachusetts, 2005.

[3] A. M. Finkelstein and V. Kreinovich, "Impossibility of hardly possible events: physical consequences", Abstracts of the 8 International Congress on Logic, Methodology, and Philosophy of Science, Moscow, 1987, Vol. 5, Part 2, pp. 23-25.

[4] U. Frisch, S. Matarrese, R. Mohayaee, and A. Sobolevsky, "A reconstruction of the initial conditions of the Universe by optimal mass transportation", Nature, 2002, Vol. 417, pp. 260-262.

[5] V. Kreinovich, "Interval computations and their possible use in estimating accuracy of the optimization-based reconstruction of the early Universe", Abstracts of the International Workshop "At the Interface of Dynamical and Statistical Cosmology and Transport Optimization", Haifa, Israel, March 22-26, 2009.

[6] V. Kreinovich and A. M. Finkelstein, "Towards Applying Computational Complexity to Foundations of Physics", Notes of Mathematical Seminars of St. Petersburg Department of Steklov Institute of Mathematics, 2004, Vol. 316, pp. 63-110; reprinted in Journal of Mathematical Sciences, 2006, Vol. 134, No. 5, pp. 2358-2382.

[7] M. Li and P. Vitányi, An introduction to Kolmogorov Complexity and its applications, Springer Verlag, New York, 2008.

[8] R. Mohayaee and A. Sobolevskii, "The Monge-Ampére-Kantorovich approach to reconstruction in cosmology", Physica D, to appear. 